\section{Longitudinal analysis of age-related changes in intraocular pressure in South Korea}

SU Baek ${ }^{1}$, C Kee ${ }^{2}$ and W Suh ${ }^{3}$

\begin{abstract}
Purpose To assess the changes in intraocular pressure (IOP) with age in South Korea Methods Subjects aged 20-79 who had been receiving health examinations at a university hospital were enrolled. They completed physical and ophthalmic examinations. Subjects with ocular disease that could possibly affect their IOP were excluded. The relationships between IOP and age, blood pressure, heart rate, body mass index, blood chemistry, and electrolyte were analyzed using a linear mixed model.

Results Of the 33712 subjects, 31857 participants were enrolled. In a cross-sectional analysis, IOP also showed a negative correlation in all age groups (respectively, $P<0.001$ ). In particular, patients in their $60 \mathrm{~s} \sim 80 \mathrm{~s}$ had a less steep decreasing slope of IOP with age than patients in their 20s $\sim 30 \mathrm{~s}$ (correlation coefficient -0.260 and -0.168 , respectively). In longitudinal analysis, negative trend was shown in the slope of tendency in total subjects. When analyzing the effect of gender on the relationship between age and IOP, females had a less steep decreasing slope of IOP with age than males by $0.05 \mathrm{~mm} \mathrm{Hg}$. With regard to systemic parameters, systolic blood pressure and heart rate were positively correlated with IOP $(P<\mathbf{0 . 0 0 1})$.

Conclusion IOP was significantly decreased with age, although the amount of change was small. In women and older age groups, IOP was less decreased than that of men and young age groups. In addition, IOP was positively associated with systolic blood pressure and heart rate.
\end{abstract}

Eye (2015) 29, 625-629; doi:10.1038/ eye.2015.11; published online 20 February 2015

\section{Introduction}

Control of IOP is the mainstay of glaucoma therapy. ${ }^{1,2}$ Most published studies have reported that the prevalence of glaucoma increases with aging. ${ }^{3}$ Therefore, it would be necessary to implement studies on changes in IOP due to aging to understand the normal distribution of IOP. There have been many studies about IOP change with aging. The results from previous studies have shown varying outcomes depending on the study design, study population selection, testing method, and other variables. There have been reported increases in IOP with increasing age in black and white populations. ${ }^{4,5}$ In a Barbados Eye Study, a population consisting of 3752 black study participants without glaucoma, age was the major factor positively associated with IOP. ${ }^{5}$ However, IOP was found to decrease with age in Japanese populations. ${ }^{6}$ Shiose and Kawase ${ }^{7}$ analysed changes in IOP in 200000 healthy subjects and concluded that IOP is physiologically maintained by a counterbalance between the IOP-lowering effects of age and the IOP-raising effects of obesity and systolic hypertension. However, studies on similar ethnic populations have not given consistent results. Nomura et $a l^{8}$ reported a cross-sectional analysis of IOP decrease according to age, and a longitudinal analysis showed that IOP increased significantly with age in both men and women.

In addition to age, many factors have been reported to influence IOP. A positive association between IOP and blood pressure, especially systolic blood pressure, has been reported in many cross-sectional studies. Also, an association between obesity and IOP has been reported. ${ }^{9,10}$ Gender also appears to have an effect on IOP. ${ }^{4,5}$ A previous study showed that in older age groups, the apparent rise in mean IOP with increasing age is greater among women than men. ${ }^{4}$ In addition, IOP was also associated with other medical problems including metabolic syndrome and chronic kidney disease in previous reports. ${ }^{11,12}$ Previous reports about age and IOP mostly employed the
${ }^{1}$ Department of Ophthalmology, Hallym University Sacred Heart Hospital, Hallym University College of Medicine, Anyang, Korea

${ }^{2}$ Department of Ophthalmology, Samsung Medical Center, Sungkyunkwan University School of Medicine, Seoul, Korea

${ }^{3}$ Department of Ophthalmology, Hallym University Dongtan Sacred Heart Hospital, Hallym University College of Medicine, Hwaseong, Korea

Correspondence: W Suh, Department of Ophthalmology, Hallym University Dongtan Sacred Heart Hospital, Hallym University College of Medicine, 40 SeokwooDong, Hwaseong, Gyeonggi-Do 445-170, Korea

Tel: +82 22639 5420; Fax: +82 226316516 . E-mail: being111@hotmail. com

Received: 15 July 2014 Accepted in revised form: 1 January 2015 Published online: 20 February 2015 
cross-sectional population design. Analyzing changes in IOP by aging using longitudinal analysis is a more appropriate method. However, longitudinal studies on the relationship between aging and IOP in South Korea are scarce. Therefore, authors evaluated the influence of aging on IOP in a healthy Korean population by both cross and longitudinal analyses.

\section{Materials and methods}

We examined healthy subjects who underwent health checkup at Hallym University Sacred Heart Hospital from June 2006 to April 2012. Approval was obtained from the Institutional Review Board/Ethics Committee. All study procedures were conducted following the tenets of the Declaration of Helsinki.

All participants underwent interviews about demographic information, medical history, and family history of medical diseases. We excluded subjects with previously diagnosed glaucoma and suspicious glaucoma who met the following criteria on fundus photos: vertical cup-to-disc $(C / D)$ ratio $\geq 0.6$; difference of the vertical $\mathrm{C} / \mathrm{D}$ ratio of $\geq 0.2$ between the eyes; violation of the ISNT rule (that the normal eye shows a characteristic configuration for the disc rim thickness of inferior $>$ superior $>$ nasal $>$ temporal); optic disc hemorrhage; and retinal nerve fiber layer defect. Subjects with best corrected visual acuity $<20 / 40$, inflammatory eye disease, ocular trauma, disease involving retina and choroid, or non-glaucomatous optic neuropathy were also excluded. IOP was measured three times in both eyes with a noncontact tonometer (Kowa KT-800, Tokyo, Japan), and IOP measurements were determined by taking the average value of three successive readings. All IOP measurements were taken by one skillful person without the application of topical anesthetic.

Systemic items such as blood pressure, heart rate, cholesterol, albumin, liver enzyme, thyroid hormone, BUN, creatine, electrolytes, chest $X$-ray, and electrocardiography were evaluated. Blood pressure was measured from the right, upper arm, in the sitting position using a mercury sphygmomanometer. Obesity was evaluated by body mass index (BMI), which was calculated by the weight divided by the height squared. We did not exclude patients taking medicine, especially antihypertensive medication, even though when hypertensive patients are started on systemic antihypertensive medication, it can influence the IOP level and the diurnal variation, postural hypotension, and nocturnal dips induced by medication, and these can further comprise ocular perfusion pressure contributing to further optic nerve hypoperfusion. ${ }^{13}$

All statistical analyses were conducted using SAS software version 9.1.3 (SAS, Inc., Cary, NC, USA).
A $P$-value of $<0.05$ was considered statistically significant. For the cross-sectional study, we used seven age groups divided by decades. Simple linear regression was used to obtain the coefficient value between age and IOP using the cross-sectional analyses. Longitudinal change in IOP was analyzed using the linear mixed model, which is a type of statistical analysis commonly used for repeated measurements. Each scatter plot of patient's IOP was analyzed respectively as time progressed, then the total aspect of change was drawn up. To understand the effect of gender on relationship between age and IOP, the two-way ANOVA test was used. Associations between IOP and various factors were analyzed in which independent variables, such as age, sex, blood pressure, heart rate, cholesterol, albumin, liver enzyme, thyroid hormone, BUN, creatine, electrolytes, chest $\mathrm{X}$-ray, and electrocardiography, were included using multiple linear regression analysis.

\section{Results}

Of the 33712 subjects, 31857 participants were enrolled. The mean age of participants was $45.6 \pm 7.7$ years. The male to female ratio was $1.83: 1$. The average IOP in all study subjects was $13.9 \pm 2.9 \mathrm{~mm} \mathrm{Hg}$ in the right eye and $13.9 \pm 3.0 \mathrm{~mm} \mathrm{Hg}$ in the left eye; the two values were not significantly different $(P=0.86)$. We selected IOP of the right eye for the study.

\section{Age-related change in IOP}

Of all the patients, $11.7 \%$ were in their $20 \mathrm{~s}$ and $30 \mathrm{~s}, 47.28 \%$ were in $40 \mathrm{~s}, 34.14 \%$ were in 50 s and $5.83 \%$ were in $60 \mathrm{~s} \sim 80 \mathrm{~s}$, showing a high proportion of $40 \mathrm{~s}$ and $50 \mathrm{~s}$ in the study population.

In the cross-sectional analysis, average IOP increased from $12.7 \mathrm{~mm} \mathrm{Hg}$ in subjects in their 20s to $14.0 \mathrm{~mm} \mathrm{Hg}$ in those in their 40s. Then IOP decreased from $13.9 \mathrm{~mm} \mathrm{Hg}$ in those in their 60s to $13.1 \mathrm{~mm} \mathrm{Hg}$ in those in their 70s. The total slope of tendency showed a positive value of 0.038 in the cross-sectional study (Figure 1). However, in the regression analysis, a negative correlation between age and IOP was shown in each age group (estimate $-0.085, P<0.001$ ) (Table 1). In particular, when analyzing the relationship between age and IOP in each age group, all age groups also showed negative estimate value (Table 2). In addition, patients over 60s had a less steep decreasing slope of IOP with age than patients under 30s (correlation coefficient -0.260 and -0.168 , respectively). When we drew a scatter plot about the relationship between age and IOP of each patient, there were variable results including negative values or positive values of slope in each measurement. However, the total analysis of all subjects, a negative trend was shown in the slope of 


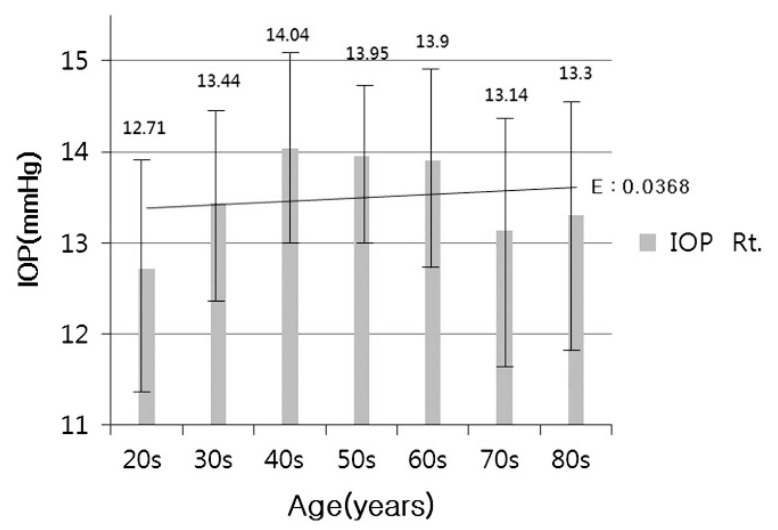

Figure 1 Changes of mean IOP according to age. For the crosssectional study, we used seven age groups classified by decades. Average IOP increased from $12.7 \mathrm{~mm} \mathrm{Hg}$ in subjects in their 20s to $14.0 \mathrm{~mm} \mathrm{Hg}$ in those in their 40s. Then IOP decreased from $13.9 \mathrm{~mm} \mathrm{Hg}$ in those in their $60 \mathrm{~s}$ to $13.1 \mathrm{~mm} \mathrm{Hg}$ in those in their $70 \mathrm{~s}$. The total slope showed a positive trend (coefficient value of $0.0368)$.

Table 1 Longitudinal analysis between IOP and other variables

\begin{tabular}{lccc}
\hline & Estimate & SE & P-value \\
\hline Age & -0.085 & 0.014 & $<0.0001$ \\
Laterality & 0.013 & 0.071 & 0.34 \\
Sex & -0.046 & 0.001 & $<0.0001$ \\
Systolic blood pressure & 0.012 & 0.001 & $<0.0001$ \\
Diastolic blood pressure & -0.003 & 0.001 & 0.05 \\
Heartbeat & 0.017 & 0.0004 & $<0.0001$ \\
Cholesterol & 0.0007 & 0.068 & 0.12 \\
Albumin & -0.159 & 0.001 & 0.02 \\
Aspartate aminotransferase & 0.013 & 0.0008 & $<0.0001$ \\
Alanine aminotransferase & -0.007 & 0.004 & $<0.0001$ \\
Thyroid-stimulating hormone & -0.004 & 0.0007 & 0.38 \\
Thyroid hormone 3 & -0.005 & 0.071 & $<0.0001$ \\
Free thyroid hormone 4 & -0.214 & 0.003 & 0.002 \\
Blood urea nitrogen & -0.013 & 0.109 & 0.0005 \\
Creatinine & 0.370 & 0.051 & 0.0007 \\
Calcium, total & 0.616 & 0.029 & $<0.0001$ \\
Phosphorus & -0.270 & 0.016 & $<0.0001$ \\
Uric acid & -0.087 & 0.084 & $<0.0001$ \\
Chest X-ray & $6.940 \sim 8.041$ & 0.047 & 1 \\
Electrocardiography & $0.023 \sim 0.152$ & & 0.001 \\
\hline
\end{tabular}

In the regression analysis, a negative correlation between age and IOP was shown in each age group (estimate $-0.085, P<0.001$ ). In the multiple regression analyses, systolic blood pressure, heartbeat, serum creatine, and total calcium $(P<0.001)$ were positively correlated with IOP $($ all $P<0.001)$. However, diastolic blood pressure and serum cholesterol were not significantly correlated with IOP change $(P=0.05, P=0.12)$.

tendency (data not shown). The scatter plot of total subjects could not be showed because so many lines overlapped. When comparing the male and female groups, IOP was still negatively correlated in both the groups (coefficient value -0.09 and -0.046 , respectively). When analyzing the effect of gender on the relationship between age and IOP, the interaction effect was 0.05 $(P<0.0001)$ (Table 3). This implies that females had a less
Table 2 The relationship between age and IOP in each age group

\begin{tabular}{lllrllll}
\hline $\begin{array}{l}\text { Age } \\
\text { group }\end{array}$ & Estimate & $S E$ & DF & $\mathrm{t}$-Value & $\operatorname{Pr}>|\mathrm{t}|$ & $\begin{array}{c}\text { Adjusted } \\
\text { P-value }\end{array}$ \\
\hline$\leq 30 \mathrm{~s}$ & -0.260 & 0.015 & 3616 & -17.25 & $<0.0001$ & $<0.0001$ \\
$40 \mathrm{~s}$ & -0.246 & 0.007 & 14000 & -34.26 & $<0.0001$ & $<0.0001$ \\
$50 \mathrm{~s}$ & -0.243 & 0.008 & 11000 & -29.89 & $<0.0001$ & $<0.0001$ \\
$60 \mathrm{~s} \geq$ & -0.168 & 0.014 & 1698 & -11.51 & $<0.0001$ & $<0.0001$ \\
\hline
\end{tabular}

Abbreviation: DF, degree of freedom.

When analyzing the relationship between age and IOP in each age group, all age groups also showed negative estimate value. In addition, patients $>60$ s had a less steep decreasing slope of IOP with age than patients under 30 s (correlation coefficient -0.260 and -0.168 , respectively).

Table 3 Effect of gender on relationship between age and IOP

\begin{tabular}{lcrccc}
\hline & Sex & Estimate & SE & t-Value & P-value \\
\hline Age & & -0.105 & 0.003 & -26.9 & $<0.0001$ \\
Sex & Female & -3.099 & 0.279 & -11.1 & $<0.0001$ \\
Age $\times$ sex & Female & 0.050 & 0.006 & 8.24 & $<0.0001$ \\
\hline
\end{tabular}

When comparing male and female groups, IOP was negatively correlated in both the groups. When analyzing the effect of gender on the relationship between age and IOP, the interaction effect was 0.05 $(P<0.0001)$. This implies that females had a less steep decreasing slope of IOP with age compared with males by $0.05 \mathrm{~mm} \mathrm{Hg}$.

steep decreasing slope of IOP with age than males by $0.05 \mathrm{~mm} \mathrm{Hg}$ (Table 3).

\section{Correlation of IOP and various factors}

In the univariable analysis, the relationship between IOP and age showed a significant negative tendency $(-0.147$, $P<0.001)$. After adjusting the confounding factor on IOP, it still showed a negative tendency (coefficient -0.085 , $P<0.001$ ) (Table 1). In the multiple regression analyses, systolic blood pressure, heartbeat, serum creatine, and total calcium $(P<0.001)$ were positively correlated with IOP (all $P<0.001$ ). However, diastolic blood pressure and serum cholesterol were not significantly correlated with the IOP change ( $P=0.05$ and 0.12 , respectively) (Table 1 ). When BMI was further evaluated, it was not found to be significantly associated with IOP change with increasing age (coefficient value $0.004, P=0.28$ ).

\section{Discussion}

IOP is known as an important factor for glaucoma pathogenesis. ${ }^{1,2}$ Therefore, it is important to identify various factors that may influence IOP. However, there is still debate about these factors, especially age. Nomura et $a l^{8}$ reported an inconsistency in changes in IOP against age between cross-sectional and longitudinal analyses, as previously mentioned. They postulated that birth cohort differences in ocular characteristics influence IOP and 
suggested that longitudinal analysis is more accurate than cross-sectional analysis in understanding age-related IOP changes. Therefore, we assessed the influence of age on IOP with both cross-sectional and longitudinal analyses by enrolling a large population. ${ }^{8}$

In the cross-sectional analysis, average IOP increased from $12.7 \mathrm{~mm} \mathrm{Hg}$ in subjects in their 20s to $14.0 \mathrm{~mm} \mathrm{Hg}$ in those in their 40s. The total slope of tendency showed a positive value of 0.038 in the cross-sectional study. However, the authors tried to control the confounding variables. And a large scale of study population itself makes statistical importance because of nature of observational study and statistical characteristics. Therefore, we divided subgroups and analyzed the correlation of age with IOP again. As a result, our study showed that age was negatively correlated with IOP (coefficient value $-0.085, P<0.001$ ) (Table 1). Previous studies have reported variable associations between age and IOP. Several studies have shown that IOP increased with age in study populations, whereas others suggested no relationship with age, after adjustment. ${ }^{5,10}$ In a Barbados Eye Study, among the black participants, the mean IOP increased $\sim 1 \mathrm{~mm} \mathrm{Hg}$ for every increase of 10 years of age. ${ }^{14}$ However, a Japanese study showed that IOP correlated inversely with age in men $(r=-0.14$, $P<0.001)$, and that IOP showed a marginal inverse correlation in women $(r=-0.07, P=0.06) .{ }^{8}$ Other study reported that IOP trended to decrease with age in all age groups, the mean linear regression coefficient in the 20s, $30 \mathrm{~s}$, and $40 \mathrm{~s}$ for 10 years was $-0.076,-0.075$, and -0.060 , respectively. ${ }^{15}$ Although all participants enrolled in the study were flight crews and young and middle age individuals, the study was well designed with long duration of follow-up. These coefficient values are similar to our results. Several possible causes of negative correlation have been suggested in previous reports. Shiose $^{16}$ suggested that the prevalence of obesity and hypertension is lower in the Japanese population than Western population, and it could affect the IOPdecreasing tendency with age. Relatively high corticosteroid secretion in obese persons from excess fat may lead to high episcleral venous pressure and decreased outflow facility. ${ }^{7,17}$ However, when we assessed the effects of BMI on IOP change with age, there was no significant difference between the high BMI group $(>23)$ and low BMI group $(\leq 23)$. Other studies have suggested that the production and outflow of aqueous humor may decrease in older individuals by accumulation of extracelluar matrix in the trabecular meshwork. ${ }^{17-19}$ In addition to the environmental effect, genetic factors may explain these differences. ${ }^{20}$

In addition, this study found that the degree of IOP change was different depending on the age group. The correlation coefficient was -0.260 in the 20 s $\sim 30$ s age groups and -0.168 in the 60 s $\sim 80$ s age group, implying that IOP of old age groups was less decreased than that of younger groups (Table 2). With regard to gender, the female group had a less steep decreasing slope of IOP with age than the male group (Table 3). Other study groups reported that men showed higher IOP than women with increasing age. ${ }^{7,15}$ However, in others, women showed higher IOP than men. Bankes et al ${ }^{21}$ reported that IOP raised steady with increasing age and was more pronounced in women than in men. Some inferred that increasing IOP was associated with postmenopause. ${ }^{22,23}$ Also, the fact that females have a higher portion of fat in body mass than males as well as hormones that are associated with postmenopause may affect IOP change due to aging. ${ }^{24}$

In addition to age and gender, many systemic factors showed correlations with IOP. Positive association between IOP and blood pressure, especially systolic blood pressure, has been reported in many cross-sectional studies. ${ }^{8,25}$ This study also showed that systolic blood pressure and heart rate have a significant positive correlation with IOP, whereas diastolic blood pressure was not significantly correlated with IOP. It has been postulated that increased blood pressure leads to elevated ciliary artery pressure, increasing the ultrafiltration of the aqueous humor and thus increasing IOP. ${ }^{9}$

There are some limitations in this study in the aspect of subject collection and the relatively short follow-up period. ${ }^{8}$ And the proportion of old age group is relatively small $(6.9 \%$ over $60 \mathrm{~s})$. It is characteristic of health screening test, and we also excluded subjects with ophthalmologic diseases, these possible cause this small proportion. However, considering the prevalence of glaucoma increases with aging, selection bias could influence the analysis results. Furthermore, our study enrolled only healthy screening subjects, therefore older participants who had hypertension, high cholesterol, and cardiovascular disease, which were highly associated with high IOP in previous literature, were selectively excluded. $8,11,12,21$ In this study, IOP was not corrected for corneal thickness and refractive error. Generally, eyes with thinner mean central corneal thickness (CCT) was known to be associated with lower IOP measurement. ${ }^{4}$ In addition, Singapore Malay Eye Study reported CCT was $547.7 \mu \mathrm{m}$ in $40 \mathrm{~s}$ and $532.3 \mu \mathrm{m}$ in $70-80$ years, applying that CCT decreased with age by $5.1 \mu \mathrm{m}$ per decade. ${ }^{12}$ In aspect with refractive error, more myopia and steeper corneal curvature were correlated with a higher IOP measurement. ${ }^{4}$ Therefore, these uncorrected factors could possibly influence on IOP distribution with age. In addition, owing to the nature of the health screening, our study used noncontact tonometer instead of the Goldmann applanation tonometer. Although previous study reported that there was no statistically significant 
difference between noncontact tonometer and the Goldmann tonometer with in the normal IOP range, variation in the values obtained with noncontact tonometer was greater than that of the Goldmann applanation tonometer, and Goldmann applanation tonometry is the gold standard for measuring IOP. ${ }^{26}$

In conclusion, IOP was significantly decreased with age, although the amount of change was small. In women and older age groups, IOP was less decreased than that of men and young age groups. In addition, IOP was positively associated with systolic blood pressure and heart rate. A further study with large study populations and longer follow-up periods will be needed in the future.

\section{Summary}

What was known before

- There have been many controversial results about IOP change with aging.

What this study adds

- In a longitudinal analysis in South Korea, IOP was significantly decreased with age, although the amount of change was small.

- In women and older age groups, IOP was less decreased than that of men and young age groups.

\section{Conflict of interest}

The authors declare no conflict of interest.

\section{References}

1 Kass MA, Heuer DK, Higginbotham EJ, Johnson CA, Keltner JL, Miller JP et al. The Ocular Hypertension Treatment Study: a randomized trial determines that topical ocular hypotensive medication delays or prevents the onset of primary open-angle glaucoma. Arch Ophthalmol 2002; 120(6): 701-713

2 Comparison of glaucomatous progression between untreated patients with normal tension glaucoma and patients with therapeutically reduced intraocular pressure. Collaborative Normal-Tension Glaucoma Study Group. Am J Ophthalmol 1998; 126(4): 487-497.

3 Mitchell P, Smith W, Attebo K, Healey PR. Prevalence of open-angle glaucoma in Australia. The Blue Mountains Eye Study. Ophthalmology 1996; 103(10): 1661-1669.

4 Klein BE, Klein R, Linton KL. Intraocular pressure in an American community. The Beaver Dam Eye Study. Invest Ophthalmol Vis Sci 1992; 33(7): 2224-2248.

5 Wu SY, Leske MC. Associations with intraocular pressure in the Barbados Eye Study. Arch Ophthalmol 1997; 115(12): 1572-1576.

6 Nakano T, Tatemichi M, Miura Y, Sugita M, Kitahara K. Long-term physiologic changes of intraocular pressure: a 10-year longitudinal analysis in young and middle-aged Japanese men. Ophthalmology 2005; 112(4): 609-616.
7 Shiose Y, Kawase T. A new approach to stratified normal intraocular pressure in a general population. Am J Ophthalmol 1986; 101(6): 714-721.

8 Nomura H, Shimokata H, Ando F, Miyake Y, Kuzuya F. Age-related changes in intraocular pressure in a large Japanese population. Ophthalmology 1999; 106(10): 2016-2022.

9 Mitchell P, Lee AJ, Wang JJ, Rochtchina E. Intraocular pressure over the clinical range of blood pressure: blue mountains eye study findings. Am J Ophthalmol 2005; 140(1): 131-132.

10 Carel RS, Korczyn AD, Rock M, Goya I. Association between ocular pressure and certain health parameters. Ophthalmology 1984; 91(4): 311-314.

11 Oh SW, Lee SY, Park CY, Kim DJ. Elevated intraocular pressure is associated with insulin resistance and metabolic syndrome. Diabetes Metab Res Rev 2005; 21: 434-440.

12 Su DH, Wong TY, Foster PJ, Tay WT, Saw SM, Aung T. Central corneal thickness and its associations with ocular and systemic factors: the Singapore Malay Eye Study. Am J Ophthalmol 2009; 147: 709-716.

13 Tielsch JM, Katz J, Sommer A, Quigley HA, Javitt JC. Hypertension, perfusion pressure, and primary open angle glaucoma: a population-based assessment. Arch Ophthalmol 1995; 113(2): 216-221.

14 Leske MC, Connell AM, Wu SY, Hyman L, Schachat AP. Distribution of intraocular pressure. The Barbados Eye Study. Arch Ophthalmol 1997; 115(8): 1051-1057.

15 Nomura H, Ando F, Niino N, Shimokata H, Miyake Y. The relationship between age and intraocular pressure in a Japanese population: the influence of central corneal thickness. Curr Eye Res 2002; 24(2): 81-85.

16 Shiose $\mathrm{Y}$. The aging effect on intraocular pressure in an apparently normal population. Arch Ophthalmol 1984; 102(6): 883-887.

17 Brubaker RF, Nagataki S, Townsend DJ, Burns RR, Higgins RG, Wentworth $W$. The effect of age on aqueous humor formation in man. Ophthalmology 1981; 88(3): 283-287.

18 Bloom JN, Levene RZ, Thomas G, Kimura R. Fluorophotometry and the rate of aqueous flow in man. I. Instrumentation and normal values. Arch Ophthalmol 1976; 94(3): 435-443.

19 Miyazaki M, Segawa K, Urakawa Y. Age-related changes in the trabecular meshwork of the normal human eye. Jpn J Ophthalmol 1987; 31(4): 558-569.

20 Weih LM, Mukesh BN, McCarty CA, Taylor HR. Association of demographic, familial, medical, and ocular factors with intraocular pressure. Arch Ophthalmol 2001; 119(6): 875-880.

21 Bankes JL, Perkins ES, Tsolakis S, Wright JE. Bedford Glaucoma Survey. Br Med J 1968; 1(5595): 791-796.

22 Altintas O, Caglar Y, Yuksel N, Demirci A, Karabaş L. The effects of menopause and hormone replacement therapy on quality and quantity of tear, intraocular pressure and ocular blood flow. Ophthalmologica 2004; 218(2): 120-129.

23 Qureshi IA. Ocular hypertensive effect of menopause with and without systemic hypertension. Acta Obstet Gynecol Scand 1996; 75(3): 266-269.

24 Toker E, Yenice O, Temel A. Influence of serum levels of sex hormones on intraocular pressure in menopausal women. J Glaucoma 2003; 12(5): 436-440.

25 Qureshi IA. Intraocular pressure: a comparative analysis in two sexes. Clin Physiol 1997; 17(3): 247-255.

26 Lee JS, Lee SH, Oum BS, Chung JS, Cho BM, Hong JW. Relationship between intraocular pressure and systemic health parameters in a Korean population. Clin Experiment Ophthalmol 2002; 30(4): 237-241. 